\title{
Ангармонизм колебаний кристаллической решетки монокристаллов $\mathrm{Bi}_{2} \mathrm{Se}_{3}$
}

\author{
() 3.И. Бадалова ${ }^{1}$, Н.А. Абдуллаев 1 , , Г.Х. Аждаров ${ }^{1}$, Х.В. Алигулиева ${ }^{1}$, \\ С.Ш. Кахраманов ${ }^{1}$, C.A. Немов ${ }^{2}$, Н.Т. Мамедов ${ }^{1}$ \\ ${ }^{1}$ Институт фризики Национальной академии наук Азербайджана, \\ Az-1143, Баку, Азербайджан \\ ${ }^{2}$ Санкт-Петербургский государственный политехнический университет Петра Великого, \\ 195251 Санкт-Петербург, Россия \\ ฯ E-mail: abnadir@mail.ru
}

(Получена 25 сентября 2018 г. Принята к печати 3 октября 2018 г.)

Изучены температурные зависимости активных в рамановском (комбинационном) рассеянии частот $E_{g}^{2}$, $A_{1 g}^{2}$ в слоистых монокристаллах $\mathrm{Bi}_{2} \mathrm{Se}_{3}$. Определен вклад теплового расширения в температурное изменение частот. Показано ослабление анизотропии упругих свойств в слоистых монокристаллах $\mathrm{Bi}_{2} \mathrm{Se}_{3}$ за счет сильного спин-орбитального взаимодействия. Вычислены модовые параметры Грюнайзена для фононов $E_{g}^{2}$ и $A_{1 g}^{2}$. Установлены закономерности в зависимостях величин частот колебаний от масс атомов в слоистых монокристаллах $\mathrm{Bi}_{2} \mathrm{Te}_{3}, \mathrm{Bi}_{2} \mathrm{Se}_{3}$ и $\mathrm{Sb}_{2} \mathrm{Te}_{3}$.

DOI: 10.21883/FTP.2019.03.47279.8987

\section{1. Введение}

Так как в реальных кристаллах потенциал взаимодействия между атомами имеет, как правило, ангармонический вид, частоты фононов $\omega$ меняются с температурой $T$. Это изменение в общем виде записывается как

$$
\frac{d \omega}{d T}=\left(\frac{\partial \omega}{\partial T}\right)_{V}+\left(\frac{\partial \omega}{\partial V}\right)_{T}\left(\frac{\partial V}{\partial T}\right)_{p},
$$

или

$$
\frac{d \omega}{d T}=\left(\frac{\partial \omega}{\partial T}\right)_{V}-\frac{\alpha}{\chi}\left(\frac{\partial \omega}{\partial p}\right) .
$$

Здесь $\alpha=\frac{1}{V}\left(\frac{\partial V}{\partial T}\right)_{p}$ - коэффициент объемного расширения, $\chi=-\frac{1}{V}\left(\frac{\partial V}{\partial p}\right)_{T}-$ коэффициент объемной сжимаемости, знак минус указывает на уменьшение объема с увеличением давления. В гармоническом приближении каждый член равенства, кроме $\chi$, равен нулю. В левой части уравнения (2) - выражение, определяющее общее изменение частоты $\omega$ с температурой. Первый член в правой части уравнения (2) отражает „явный“ (,explicit"،) вклад фонон-фононного взаимодействия при фиксированном положении равновесия атомов и значений силовых постоянных. Второй член, так называемый „неявный“ („,implicit“) вклад, отражает влияние на величины частот изменения межатомного расстояния и силовых постоянных с температурой вследствие теплового расширения.

Соотношение первого и второго членов в выражении (2) представляет большой интерес и является необходимым для понимания влияния температуры на фононный спектр кристалла. Например, в квазигармоническом приближении полностью доминирует второй член в (2). В работе [1] оценен вклад теплового расширения в общее изменение частот с температурой в кристаллах с ковалентной связью и молекулярных кристаллах с двумя видами связи в элементарной ячейке: ковалентной („внутримолекулярной“ связью) и типа ван-дер-Ваальса („межмолекулярной“ связью). Показано, что малый вклад теплового расширения характерен для ковалентных кристаллов и „внутримолекулярных“ колебаний в молекулярных кристаллах. В [2] также выявлено, что вклад теплового расширения в температурную зависимость частот активных в рамановском (комбинационном) рассеянии (КР-активных) фононов в слоистых кристаллах $\mathrm{GaS}$ существенно зависит от характера связей атомов в кристаллической решетке. Для фононов, частота которых определяется слабой межслоевой связью типа ван-дер-Ваальса, в основном (на 75\%) изменение частоты фонона с температурой обусловлено вкладом теплового расширения. Изменение же с температурой частоты фононов, определяемых сильной внутрислоевой связью, главным образом обусловлено вкладом первого члена в выражении (2). В настоящей работе нами проанализирована роль каждого слагаемого (2) в общем изменении частот КР-активных фононов $\omega$ в слоистых монокристаллах $\mathrm{Bi}_{2} \mathrm{Se}_{3}$ с температурой, вычислены значения модовых параметров Грюнайзена.

\section{2. Эксперимент}

Монокристаллы $\mathrm{Bi}_{2} \mathrm{Se}_{3}$ выращивались методом Бриджмена и вертикальной направленной кристаллизацией из компонент со стехиометрическим соотношением. Направленная зонная кристаллизация проводилась при скорости перемещения зоны $3 \mathrm{~cm} /$ ч. Рентгенструктурные исследования образцов проводились на рентгеновском дифрактометре Bruker D8 Advance. Однородность образцов и характерные частоты активных в рамановском рассеянии фононов исследовались на рамановском конфокальном микроспектрометре 


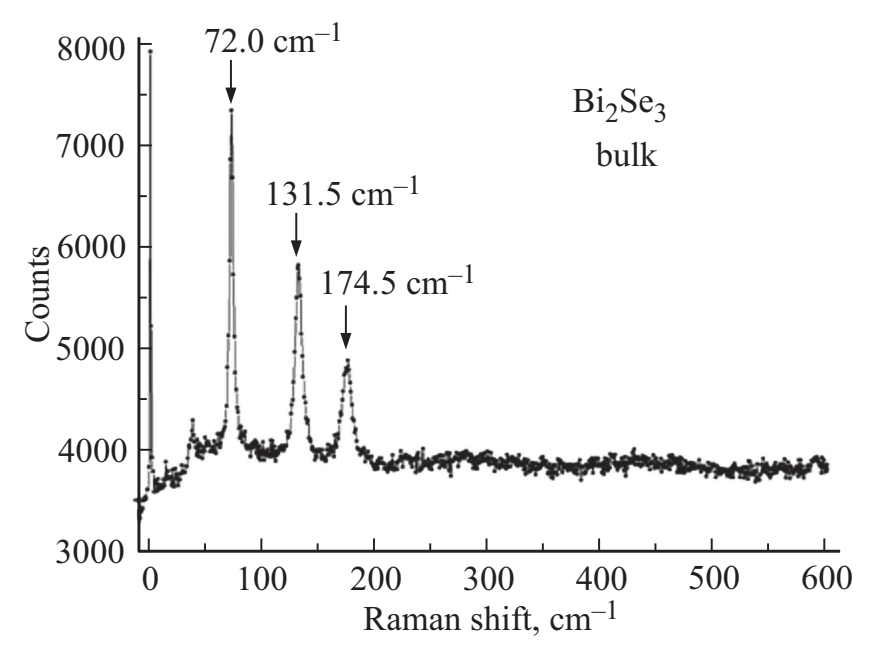

Pис. 1. Спектр комбинационного рассеяния света в монокристаллах $\mathrm{Bi}_{2} \mathrm{Se}_{3}$ при температуре $T=300 \mathrm{~K}$.

Nanofinder 30 (Tokyo Instr., Japan). Измерения проводились в геометрии обратного рассеяния. В качестве источника возбуждающего света использовался лазер YAG : Nd c длиной волны излучения на второй гармонике $\lambda=532$ нм, мощность падающего на образец излучения 7-9 мВт, диаметр луча $\sim 4$ мкм. Приемником излучения служила охлаждаемая $\mathrm{CCD}$-камера $\left(-70^{\circ} \mathrm{C}\right)$, работающая в режиме счета фотонов. В спектрометре использовалась дифракционная решетка 1800 штрихов/мм, при этом точность определения спектрального положения линий была не хуже $0.5 \mathrm{~cm}^{-1}$.

Характерный спектр комбинационного рассеяния света в объемных монокристаллах $\mathrm{Bi}_{2} \mathrm{Se}_{3}$ при комнатной температуре приведен на рис. 1. В спектре отчетливо видны три спектральные линии с максимумами при частотах $72,131.5$ и $174.5 \mathrm{~cm}^{-1}$.

\section{3. Частоты комбинационного рассеяния в монокристаллах $\mathrm{Bi}_{2} \mathrm{Se}_{3}$}

Как известно, в элементарной ячейке $\mathrm{Bi}_{2} \mathrm{Se}_{3}$ имеется 5 атомов и соответственно 15 решеточных колебательных мод в центре зоны Бриллюэна (при $q=0), 3$ из которых являются акустическими и 12 - оптическими

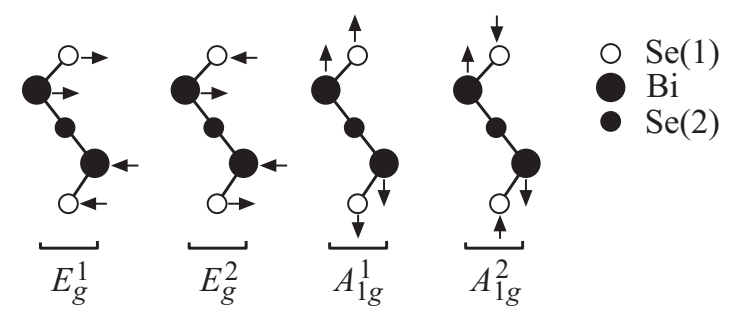

Рис. 2. Смещения атомов в элементарной ячейке кристаллов $\mathrm{Bi}_{2} \mathrm{Se}_{3}$ для каждой КР-активной моды $E_{g}^{1}, A_{1 g}^{1}, E_{g}^{2}, A_{1 g}^{2}$.
Таблица 1. Величины частот (в см $\left.{ }^{-1}\right)$ КР-активных $\left(E_{g}^{1}, A_{1 g}^{1}\right.$, $\left.E_{g}^{2}, A_{1 g}^{2}\right)$ и ИК-активных $\left(E_{u}^{1}, A_{1 u}^{1}, E_{u}^{2}, A_{1 u}^{2}\right)$ мод в монокристаллах $\mathrm{Bi}_{2} \mathrm{Se}_{3}, \mathrm{Bi}_{2} \mathrm{Te}_{3}$ и $\mathrm{Sb}_{2} \mathrm{Te}_{3}[3]$

\begin{tabular}{l|l|l|l|l|l|l|l|r}
\hline Состав & $A_{1 g}^{1}$ & $A_{1 g}^{2}$ & $E_{g}^{1}$ & $E_{g}^{2}$ & $A_{1 u}^{1}$ & $A_{1 u}^{2}$ & $E_{u}^{1}$ & $E_{u}^{2}$ \\
\hline $\mathrm{Bi}_{2} \mathrm{Se}_{3}$ & 72 & 174.5 & - & 131.5 & - & - & 65 & 129 \\
$\mathrm{Bi}_{2} \mathrm{Te}_{3}$ & 62.5 & 134 & - & 103 & 94 & 120 & 50 & 95 \\
$\mathrm{Sb}_{2} \mathrm{Te}_{3}$ & 69 & 165 & - & 112 & & & &
\end{tabular}

модами. 12 оптических мод характеризуются симметрией $2 E_{g}+2 A_{1 g}+2 E_{u}+2 A_{1 u}$, каждая из мод $E_{g}$ и $A_{1 g}$ дважды вырождены. В каждом квинтете, т.е. слое $\mathrm{Se}(1)-\mathrm{Bi}-\mathrm{Se}(2)-\mathrm{Bi}-\mathrm{Se}(1)-$, при колебаниях $E_{g}^{1}, E_{g}^{2}$ смещения атомов происходят перпендикулярно оси с оси симметрии 3-го порядка, т.е. в плоскости слоев, а при колебаниях $A_{1 g}^{1}, A_{1 g}^{2}$ смещения атомов происходят соответственно параллельно оси с, т. е. перпендикулярно плоскости слоев (рис. 2).

Спектры комбинационного рассеяния (КР) и инфракрасного (ИК) отражения бинарных объемных монокристаллов $\mathrm{Bi}_{2} \mathrm{Te}_{3}, \mathrm{Bi}_{2} \mathrm{Se}_{3}$ и $\mathrm{Sb}_{2} \mathrm{Te}_{3}$ хорошо изучены теоретически и экспериментально [3]. Соответствующие значения частот КР- и ИК-активных мод при температуpe $T=300 \mathrm{~K}$ для соединения $\mathrm{Bi}_{2} \mathrm{Se}_{3}$ приведены в табл. 1 .

Отметим, что в халькогенидных слоистых кристаллах две самые низкие частотные моды $E$ и $A$ связаны с колебаниями атомов соседних слоев относительно друг друга. Мода $E_{g}^{1}$ - это колебания, при которых происходит сдвиг соседних слоев относительно друг друга, а мода $A_{1 g}^{1}$ - это колебания, при которых происходят колебания одного слоя относительно другого вдоль оси с (рис. 2). Также из рис. 2 следует, что в модах $E_{g}^{1}$ и $A_{1 g}^{1}$ колебания пар атомов $\mathrm{Se}(1)-\mathrm{Bi}$ синфазны и потому в основном напряжены связи $\mathrm{Bi}-\mathrm{Se}(2)$. В этом случае в приближении линейной цепочки можно считать массу равной сумме масс атомов $\mathrm{Bi}$ и $\mathrm{Se}: M=m_{\mathrm{Bi}}+m_{\mathrm{Se}}$. Поскольку расстояния между атомами $d(\mathrm{Bi}-\mathrm{Se}(2))=3.06 \AA$ больше, чем расстояния $d(\mathrm{Se}(1)-\mathrm{Bi})=2.99 \AA \quad[3]$, можно предположить, что силовые постоянные связи $\mathrm{Bi}-\mathrm{Se}(2)$ меньше, чем связи $\mathrm{Se}(1)-\mathrm{Bi}$. Вследствие этого и межслоевого характера колебаний моды $E_{g}^{1}$ и $A_{1 g}^{1}$ имеют, как и ожидается, более низкие частоты, чем моды $E_{g}^{2}$ и $A_{1 g}^{2}$. Две другие КР-активные моды, $E_{g}^{2}$ и $A_{1 g}^{2}$, обусловлены внутрислоевыми противофазными смещениями атомов $\mathrm{Se}(1)-\mathrm{Bi}$ под воздействием сильных ковалентных связей и имеют более высокую частоту (рис. 2). В этом случае используется приведенная масса $\mu: 1 / \mu=1 / m_{\mathrm{Bi}}+1 / m_{\mathrm{Se}}$. Воспользуемся для частот мод $E$ и $A$ соотношением

$$
v_{E, A}^{2}=\frac{k}{M} .
$$

Это соотношение имеет сильно упрощенный вид, но правильно отображает характерные особенности частот для изоструктурных кристаллов группы $\mathrm{A}_{2}^{\mathrm{V}} \mathrm{B}_{3}^{\mathrm{VI}}$. На рис. 3 


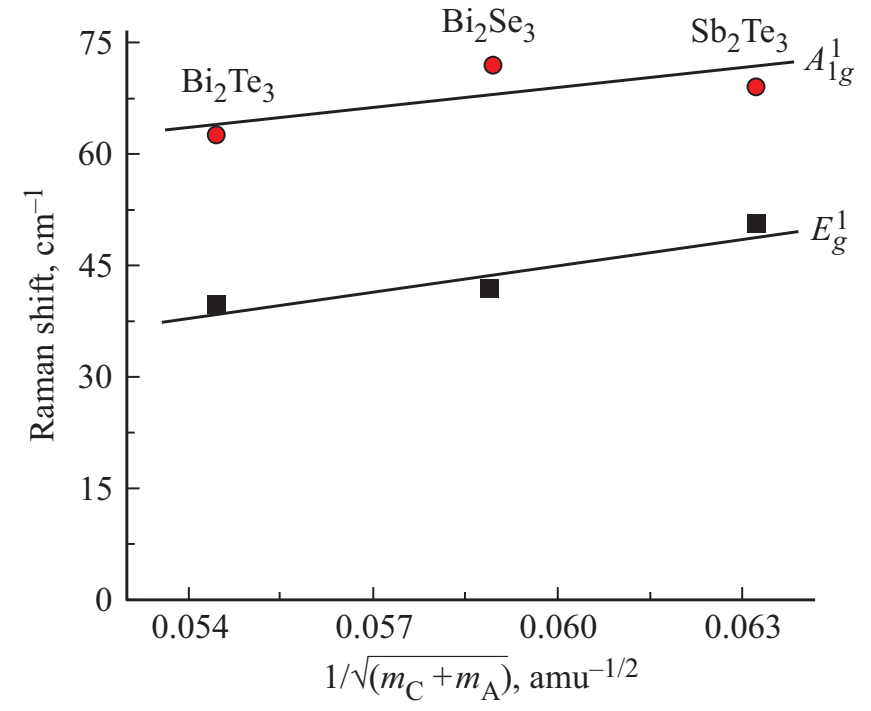

Рис. 3. Зависимости частот мод $E_{g}^{1}$ и $A_{1 g}^{1}$ от массы $M=m_{C}+m_{A}$ соединений $\mathrm{Bi}_{2} \mathrm{Te}_{3}, \mathrm{Bi}_{2} \mathrm{Se}_{3}$ и $\mathrm{Sb}_{2} \mathrm{Te}_{3}\left(m_{C}-\right.$ массы катионов $\mathrm{Bi}, \mathrm{Sb}, m_{A}-$ массы анионов Te, $\mathrm{Se}$ соответственно).

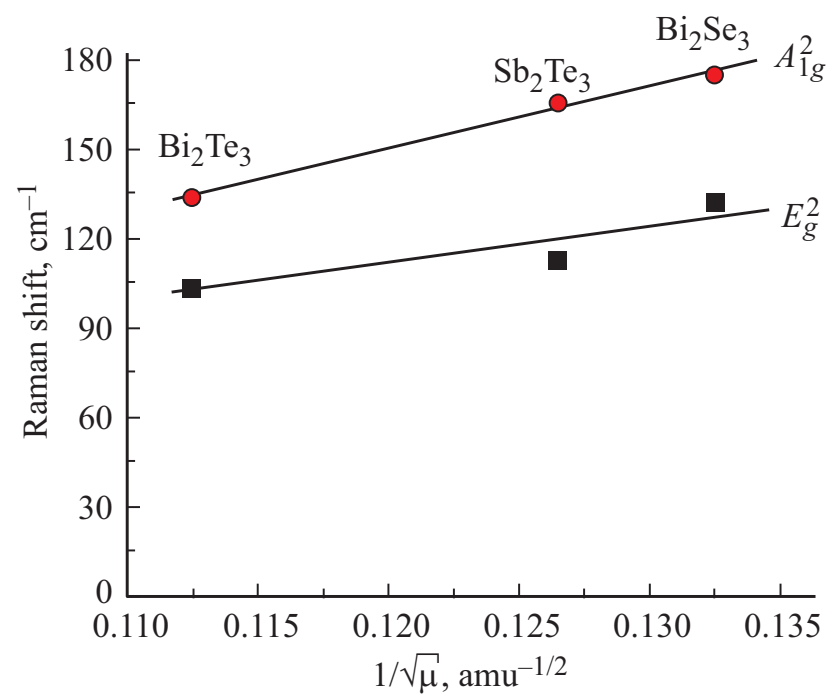

Рис. 4. Зависимости частот мод $E_{g}^{2}$ и $A_{1 g}^{2}$ от приведенной массы $\mu\left(1 / \mu=1 / m_{C}+1 / m_{A}\right)$ соединений $\mathrm{Bi}_{2} \mathrm{Te}_{3}, \mathrm{Bi}_{2} \mathrm{Se}_{3}$ и $\mathrm{Sb}_{2} \mathrm{Te}_{3}$.

и 4 приведены зависимости частот мод $E_{g}^{1}, A_{1 g}^{1}$ (рис. 3) и мод $E_{g}^{2}, A_{1 g}^{2}$ (рис. 4) от масс $M$ и $\mu$ соответственно для соединений $\mathrm{Bi}_{2} \mathrm{Te}_{3}, \mathrm{Bi}_{2} \mathrm{Se}_{3}$ и $\mathrm{Sb}_{2} \mathrm{Te}_{3}$. Величины частот мод $E_{g}^{1}$ для всех кристаллов взяты из работы [4]. Как видно из рисунков, наблюдается определенная закономерность в зависимостях частот КР-активных фононов от масс в соединениях группы $\mathrm{A}_{2}^{\mathrm{V}} \mathrm{B}_{3}^{\mathrm{VI}}$.

\section{4. Изменение частот фононов с температурой}

Нами были исследованы температурные зависимости КР-активных частот $E_{g}^{2}\left(131.5 \mathrm{~cm}^{-1}\right)$ и $A_{1 g}^{2}\left(174.5 \mathrm{~cm}^{-1}\right)$ в объемных монокристаллах $\mathrm{Bi}_{2} \mathrm{Se}_{3}$. Температурные зависимости частот приведены на рис. 5. Как видно из рис. 5 , относительное изменение частоты $\Delta \omega / \omega$ моды $E_{g}^{2}$ почти в 2 раза выше, чем $A_{1 g}^{2}$.

Согласно работам [4,5], в объемных монокристаллах $\mathrm{Bi}_{2} \mathrm{Se}_{3}$ изменение с давлением частот, $\partial \omega / \partial p$, фононов $E_{g}^{2}$ и $A_{1 g}^{2}$ в области давлений $0-4$ ГПа при комнатной температуре равно 4.1 и $2.9 \mathrm{~cm}^{-1} /$ ГПа соответственно. В работе [4] из данных по исследованию рентгеновской дифракции при гидростатическом давлении образцов $\mathrm{Bi}_{2} \mathrm{Se}_{3}$ получено значение объемного модуля упругости (bulk modulus) $B=-V\left(\frac{\partial p}{\partial V}\right)$, оказавшееся равным 53 ГПа.

Для оценок по соотношениям (1) и (2) необходимо знание коэффициента объемного расширения $\alpha$. В работе [6] приведены данные исследований температурной зависимости параметров решетки монокристаллов $\mathrm{Bi}_{2} \mathrm{Se}_{3}$ методом дифракции рентгеновских лучей. Выявлена значительная анизотропия теплового расширения в $\mathrm{Bi}_{2} \mathrm{Se}_{3}$, характерная для слоистых кристаллов [7] - при комнатной температуре величина коэффициента линейного расширения вдоль слабой связи $\beta_{\|}=1.9 \cdot 10^{-5} \mathrm{~K}^{-1}$ (паралельно тригональной оси с) значительно превышает величину коэффициента линейного расширения вдоль сильной связи $\beta_{\perp}=1.1 \cdot 10^{-5} K^{-1}$ (перпендикулярно тригональной оси с), почти в 2 раза. С учетом аксиальной симметрии кристаллов $\mathrm{Bi}_{2} \mathrm{Se}_{3}$, величина коэфициента объемного расширения равна $\alpha=\beta_{\|}+2 \beta_{\perp}=4.1 \cdot 10^{-5} \mathrm{~K}^{-1}$.

Результаты вычислений вклада теплового расширения (TE) и фонон-фононного взаимодействия (PРI) в общее изменение величин (total) КР-активных частот $E_{g}^{2}$ $\left(131.5 \mathrm{~cm}^{-1}\right)$ и $A_{1 g}^{2}\left(174.5 \mathrm{~cm}^{-1}\right)$ с изменением температуры в кристаллах $\mathrm{Bi}_{2} \mathrm{Se}_{3}$ приведены в табл. 2. Параметр $\eta$ определяет долю вклада теплового расширения (TE) в общее изменение величин частот (total).

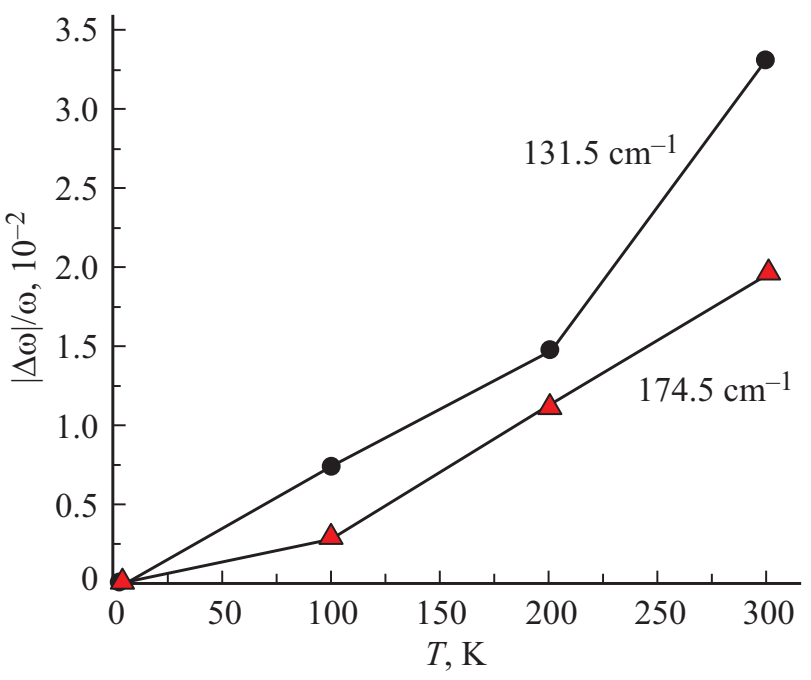

Рис. 5. Температурные зависимости КР-активных мод $E_{g}^{2}$ $\left(131.5 \mathrm{~cm}^{-1}\right)$ и $A_{1 g}^{2}\left(174.5 \mathrm{~cm}^{-1}\right)$ в кристаллах $\mathrm{Bi}_{2} \mathrm{Se}_{3}$. 
Таблица 2. Вклад теплового расширения (ТE) и фонон-фононного взаимодействия (PPI) в общее изменение величин (total) КР-активных частот $E_{g}^{2}\left(131.5 \mathrm{~cm}^{-1}\right)$ и $A_{1 g}^{2}\left(174.5 \mathrm{~cm}^{-1}\right)$ в кристаллах $\mathrm{Bi}_{2} \mathrm{Se}_{3}$

\begin{tabular}{c|c|c|c|c|c|c|c}
\hline $\begin{array}{c}\omega, \mathrm{cm}^{-1} \\
(300 \mathrm{~K})\end{array}$ & $\begin{array}{c}\partial \omega / \partial p, \\
\mathrm{~cm}^{-1} / \Gamma \Pi \mathrm{a}\end{array}$ & $\begin{array}{c}B, \\
\Gamma \Pi \mathrm{a}\end{array}$ & $\begin{array}{c}\alpha, \\
10^{-5} \mathrm{~K}^{-1}\end{array}$ & $\begin{array}{c}10^{3}|\Delta \omega| / \omega \\
(\text { total })\end{array}$ & $\begin{array}{c}10^{3}|\Delta \omega| / \omega \\
(\mathrm{TE})\end{array}$ & $\begin{array}{c}10^{3}|\Delta \omega| / \omega \\
(\mathrm{PPI})\end{array}$ & $\eta$ \\
\hline 131.5 & 4.1 & 53 & 4.1 & 33.1 & 19.6 & 13.5 & 0.59 \\
174.5 & 2.9 & 53 & 4.1 & 19.6 & 10.6 & 9 & 0.54
\end{tabular}

Выше отмечалось, что для ковалентных кристаллов и „внутримолекулярных“ колебаний в молекулярных кристаллах характерен малый вклад теплового расширения в температурное изменение частот, $\eta$ не превышает значения 0.3 [1]. В [2] вклад теплового расширения в температурное изменение частот внутрислоевых колебаний в слоистых кристаллах $\mathrm{GaS}$ составляет $\eta=0.4$. Из табл. 2 следует, что в кристаллах $\mathrm{Bi}_{2} \mathrm{Se}_{3}$ вклад теплового расширения в температурное изменение частот внутрислоевых колебаний с ковалентными связями $E_{g}^{2}$ и $A_{1 g}^{2}$ превышает 0.5. Возможно, это связано с тем, что анизотропия сил связей в $\mathrm{Bi}_{2} \mathrm{Se}_{3}$ значительно меньше, чем в слоистом $\mathrm{GaS}$, вследствие наличия сильного спин-орбитального взаимодействия, отсутствующего в кристаллах типа GaS. B работах $[8,9]$ показано, что при расчетах из первых принципов учет спин-орбитального взаимодействия увеличивает межслоевые силы в $\mathrm{Bi}_{2} \mathrm{Se}_{3}$, что отражается в уменьшении расстояния между слоями. Без учета спин-орбитального взаимодействия расстояние между атомами $\mathrm{Se}(1)-\mathrm{Se}(1)$ составляет $3.35 \AA$, а с учетом - $3.27 \AA$. В $[10,11]$ сообщается о слабой анизотропии упругих постоянных в $\mathrm{Bi}_{2} \mathrm{Se}_{3}$. Отношение величины упругой постоянной $c_{11}$, характеризующей внутрислоевую связь, к величине упругой постоянной $c_{33}$, характеризующей межслоевую связь в $\mathrm{Bi}_{2} \mathrm{Se}_{3}$, мало: $\sim 1.31$ в [10] и 1.15 в [11], для сравнения - в $\mathrm{GaS}$ отношение $c_{11} / c_{33} \approx 3.2[12]$.

\section{5. Модовые параметры Грюнайзена}

Мерой ангармоничности сил, действующих в кристалле, являются параметры Грюнайзена. Параметры Грюнайзена являются одними из важнейших характеристик динамики кристаллической решетки. Они входят в уравнение состояния, отражают особенности и характер распределения частот фононного спектра и их изменения при приложении давления. Величины параметров

Таблица 3. Величины модовых параметров Грюнайзена $\gamma_{j}$ для мод $E_{g}^{2}$ и $A_{1 g}^{2}$ в монокристаллах $\mathrm{Bi}_{2} \mathrm{Se}_{3}$

\begin{tabular}{c|c|c|c|c}
\hline Мода & $\omega_{j}, \mathrm{cм}^{-1}(300 \mathrm{~K})$ & $\partial \omega_{j} / \partial p, \mathrm{~cm}^{-1} /$ Га & $B$, ГПа & $\gamma_{j}$ \\
\hline$E_{g}^{2}$ & 131.5 & 4.1 & 53 & 1.65 \\
$A_{1 g}^{2}$ & 174.5 & 2.9 & 53 & 0.88
\end{tabular}

Грюнайзена определяют такие физические процессы, как тепловое расширение, теплопроводность, поглощение звука и др.

Величины модовых параметров Грюнайзена могут отличаться не только по величине, но и по знаку [13]. Модовые параметры Грюнайзена отражают реакцию характерных частот колебаний на деформацию кристалла и для $j$-й моды равны:

$$
\gamma_{j}=-\frac{\partial \ln \omega_{j}}{\partial \ln V}=\frac{1}{\omega_{j}} \frac{\partial \omega_{j}}{\partial p} B .
$$

Здесь $B$ - объемный модуль упругости, равный в кристаллах $\mathrm{Bi}_{2} \mathrm{Se}_{3} 53$ ГПа [4].

Вычисленные из (4) величины модовых параметров Грюнайзена для мод $E_{g}^{2}$ и $A_{1 g}^{2}$ представлены в табл. 3.

\section{6. Заключение}

Из исследований температурной зависимости КРактивных фононов в слоистых кристаллах $\mathrm{Bi}_{2} \mathrm{Se}_{3}$ выявлено, что вклад теплового расширения в изменение внутрислоевых частот $E_{g}^{2}$ и $A_{1 g}^{2}$ с температурой достаточно велик, превышает 50\%. Из этого следует, что анизотропия сил связей в $\mathrm{Bi}_{2} \mathrm{Se}_{3}$ намного меньше, чем в других халькогенидных слоистых кристаллах, например, в $\mathrm{GaS}$ и GaSe. Предполагается, что это обусловлено сильным спин-орбитальным взаимодействием в кристаллах $\mathrm{Bi}_{2} \mathrm{Se}_{3}$, отсутствующим в слоистых кристаллах типа $\mathrm{GaS}$, вносящим свой вклад в межслоевое взаимодействие. Данные об анизотропии упругих свойств кристаллов $\mathrm{Bi}_{2} \mathrm{Se}_{3}$ подтверждают это предположение.

Выявлена закономерность в зависимости величин частот КР-активных фононов в монокристаллах $\mathrm{Bi}_{2} \mathrm{Te}_{3}$, $\mathrm{Bi}_{2} \mathrm{Se}_{3}$ и $\mathrm{Sb}_{2} \mathrm{Te}_{3}$ от масс атомов. Показано, что при синфазном колебании пар атомов $\mathrm{Se}(1)-\mathrm{Bi}$ в приближении линейной цепочки для низкочастотных мод $E_{g}^{1}$ и $A_{1 g}^{1}$ можно считать массу равной сумме масс атомов, $M=m_{\mathrm{Bi}}+m_{\mathrm{Se}}$, а при противофазных колебаниях для высокочастотных мод $E_{g}^{2}$ и $A_{1 g}^{2}$ масса равна приведенной мacce $\mu\left(1 / \mu=1 / m_{\mathrm{Bi}}+1 / m_{\mathrm{Se}}\right)$.

Вычислены модовые параметры Грюнайзена $\gamma_{j}$ для мод $E_{g}^{2}$ и $A_{1 g}^{2}$.

Работа выполнена при финансовой поддержке Фонда развития науки при президенте Азербайджанской республики (гранты № EIF/MQM/Elm-Tehsil-1-2016-1(26)71/16/1 и № EİF-BGM-3-BRFTF-2'/2017-15/02/1). 


\section{Список литературы}

[1] R. Zallen, E.M. Conwell. Sol. St. Commun., 31, 557 (1979).

[2] N.A. Abdullaev, L.N. Alieva, R.A. Suleimanov. Phys. Status Solidi B, 129, K13 (1985).

[3] W. Richter, H. Kohler, C.R. Becker. Phys. Status Solidi B, 84, 619 (1977).

[4] R. Vilaplana, D. Santamaria-Perez, O. Gomis, F.J. Manjon, J. Gonzalez, A. Segura, A. Munoz, P. Rodriguez-Hernandez, E. Perez-Gonzalez, V. Marın-Borras, V. Munoz-Sanjose, C. Drasar, V. Kucek. Phys. Rev. B, 84, 184110 (2011).

[5] F.J. Manjon, R. Vilaplana, O. Gomis, E. Perez-Gonzalez, D. Santamaria-Perez, V. Marin-Borras, A. Segura, J. Gonzalez, P. Rodriguez-Hernandez, A. Munoz, C. Drasar, V. Kucek, V. Munoz-Sanjose. Phys. Status Solidi B, 250, 669 (2013).

[6] X. Chen, H.D. Zhou, A. Kiswandhi, I. Miotkowski, Y.P. Chen, P.A. Sharma, A.L. Lima Sharma, M.A. Hekmaty, D. Smirnov, Z. Jiang. Appl. Phys. Lett., 99, 261912 (2011).

[7] G.L. Belenkii, E.Yu. Salaev, R.A. Suleimanov, N.A. Abdullaev, V.Ya. Shteinshraiber. Sol. St. Commun., 53, 967 (1985).

[8] W. Cheng, S. Ren. Phys. Rev. B, 83, 094301 (2011).

[9] B.T. Wang, P. Zhang. Appl. Phys. Lett., 100, 082109 (2012).

[10] X. Gao, M. Zhou, Y. Cheng, G. Ji. Phil. Mag., 96, 208 (2016).

[11] H. Koc, H. Ozisik, E. Deligöz, A.M. Mamedov, E. Ozbay. J. Molec. Model., 20, 2180 (2014).

[12] M. Gatulle, M. Fischer, A. Chevy. Phys. Status Solidi B, 119, 327 (1983).

[13] Н.А. Абдуллаев. ФТТ, 43, 697 (2001).

Редактор Л.В. Шаронова

\section{Anharmonicity lattice vibrations of $\mathrm{Bi}_{2} \mathrm{Se}_{3}$ single crystals}

Z.I. Badalova1, N.A. Abdullayev', G.H. Azhdarov', Kh.V. Aliguliyeva ${ }^{1}$, S.Sh. Gahramanov' ${ }^{1}$ S.A. Nemov ${ }^{2}$, N.T. Mamedov ${ }^{1}$

${ }^{1}$ Institute of Physics, Azerbaijan National Academy of Sciences, Az-1143 Baku, Azerbaijan 2 Peter the Great St. Petersburg Polytechnic University, 195251 St. Petersburg, Russia

Abstract The temperature dependences of Raman-active frequencies $E_{g}^{2}, A_{1 g}^{2}$ in layered $\mathrm{Bi}_{2} \mathrm{Se}_{3}$ single crystals have been studied. The contributions of thermal expansion into temperature change of frequencies are defined. Weakening of the anisotropy of elastic properties in $\mathrm{Bi}_{2} \mathrm{Se}_{3}$ layered single crystals are due to the strong spin-orbital interaction. The Gruneisen mode parameters for phonons $E_{g}^{2}$ and $A_{1 g}^{2}$ are calculated. The regularities in the dependences of the values of the oscillation frequency on the masses of the atoms in the layered $\mathrm{Bi}_{2} \mathrm{Te}_{3}, \mathrm{Bi}_{2} \mathrm{Se}_{3}$, and $\mathrm{Sb}_{2} \mathrm{Te}_{3}$ single crystals was established. 